\title{
An Improved Bird Swarm Algorithm with Adaptive Characteristics
}

\author{
Chao Zhou ${ }^{a}$, Lei Mei ${ }^{b}$, Ryad Chellali ${ }^{c}$, Yongkun Zhao ${ }^{d}$ \\ School of Nanjing Tech University, Nanjing211816, China. \\ a1435677124@qq.com, bmeilei@njtech.edu.cn, crchellali@njetech.edu.cn, ${ }^{d} 1486230798 @ q q . c o m$
}

\begin{abstract}
Bird swarm algorithm (BSA) is a new heuristic intelligent algorithm, which has been successfully applied in many fields. In view of the shortcomings of bird swarm algorithm which is easy to fall into local optimum and premature convergence. I propose an improved bird swarm algorithm (IBSA). Firstly, the initial population is constructed by chaos optimization algorithm, so that the initial solution is uniformly distributed in the solution space, thus improving the diversity of the population. Secondly, by introducing inertial weights, nonlinear adjustment of cognitive and social coefficients can be trade-off bird local and global search ability. Finally, a disturbance strategy is added to the forging position of the birds. Thus, the diversity of population in the late iteration period is enhanced, and the ability to jump out of the local optimum is improved. Through the simulation experiments of several benchmark functions and compared with other intelligent algorithms, the results show that the improved bird swarm algorithm (IBSA) has better convergence speed and optimization precision, which proves its superiority.
\end{abstract}

Keywords: bird swarm algorithm, inertial weight, disturbance strategy, simulation test.

\section{Introduction}

In recent years, the meta-heuristic swarm intelligence algorithm has made great progress. It is a stochastic optimization algorithm and inspired by natural phenomena or animal behavior in nature [1]. The essence of the algorithm is to establish the objective function of the problem, then find the optimal solution, at last solve the actual engineering optimization problem. There is no need to optimize the problem continuous, differentiable and other conditions [2], thus, swarm intelligence optimization algorithm has wide applicability, and becomes one of the research hotspots in the field of optimization. Since 1990s, a series of swarm intelligence optimization algorithms were designed, such as ant colony algorithm (ACO) [3], particle swarm optimization (PSO) [4], gravitational search algorithm (GSA) [5] and so on.

Bird swarm algorithm (BSA) was proposed by Meng [6] in 2015. It is a new stochastic optimization algorithm developed through the information sharing mechanism and search strategy in the foraging process of birds. Compared with other swarm intelligence algorithms, it has fewer adjusting parameters, faster convergence speed and stronger robustness. The bird swarm algorithm has been successfully applied to multi-objective optimization of micro grids [7], optimal operation of cascade reservoirs [8] and flexible job shop scheduling [9]. Although the BSA as well as other traditional swarm intelligence algorithms have good precision and global optimization, there still exist some problems, such as it has poor initial population and is easy to fall into the local optimal [10].

Our contribution is organized as follows: Section2 introduces the basic bird swarm algorithm. Section3 introduces the improved bird swarm algorithm. Section4 is the experimental result. Section5 is the summary.

\section{Fundamental Theories}

\subsection{Basic Bird Swarm Algorithm}

Bird swarm algorithm is a biological heuristic algorithm derived from bird foraging, vigilance and flight behavior in nature. 
Foraging behavior: Each bird feeds food on the basis of personal experience or group experience. If a random number uniformly distributed between $(0,1)$, then the bird would forage for food. Otherwise, the bird would still vigilant. As shown by formula (1):

$$
x_{i, j}^{t+1}=x_{i, j}^{t}+\left(p_{i, j}-x_{i, j}^{t}\right) \cdot C \cdot \operatorname{rand}(0,1)+\left(g_{j}-x_{x, j}^{t}\right) \cdot S \cdot \operatorname{rand}(0,1)
$$

Where $x_{i, j}^{t}$ represents the $j$ th dimensional position of the ith bird in the tth generation population. $j \in[1, \ldots D], C$ and $S$ are learning coefficient, which can be respectively called as cognitive and social accelerated coefficient. rand $(0,1)$ denotes independent uniformly distributed numbers in $(0,1), g_{j}$ is the best previous position shared by the swarm and $p(i, j)$ is the best previous position of the bird.

Vigilance behavior: Birds will try to move to the center of the group, and they would inevitably compete with each other. Their behavior can be described by the following formulas:

$$
\begin{gathered}
x_{i, j}^{t+1}=x_{i, j}^{t}+A 1\left(\text { mean }_{j}-x_{i, j}^{t}\right) \cdot \operatorname{rand}(0,1)+A 2\left(p_{k, j}-x_{i, j}^{t}\right) \cdot \operatorname{rand}(-1,1) \\
A 1=a 1 \cdot \exp \left(-\frac{p F i t_{i}}{\text { sumFit }+\varepsilon} \cdot N\right) \\
A 2=a 2 \cdot \exp \left[\left(\frac{p_{\text {Fiti }}-p_{\text {Fiti }}}{\left|p_{\text {Fit }}-p_{\text {Fit }}\right|+\varepsilon} \cdot N\right) \frac{N \cdot p_{\text {Fitk }}}{\text { sumFit }+\varepsilon}\right]
\end{gathered}
$$

Where $k(k \neq i)$ is a positive integer, which is randomly selected between 1 and N. $a_{1}$ and $a_{2}$ are two positive constants in $[0,2]$. sumFit means the sum of the swarm's best fitness value. $p F i t_{i}$ denotes the ith bird's best fitness value. $\varepsilon$ is the smallest constant in the computer, in order to avoid zero-division error. mean $_{j}$ represents the element of the average position of the whole bird's swarm.

Flight behavior: Due to the threat of predator or other reasons, birds will fly to another place to search for food. Some birds acting as producer, but the others want to get food from producers. According to Rule (4), the behavior of producers and scroungers can be described in mathematical way, which is as follows:

$$
\begin{gathered}
x_{i, j}^{t+1}=x_{i, j}^{t}+\operatorname{randn}(0,1) \times x_{i, j}^{t} \\
x_{i, j}^{t+1}=x_{i, j}^{t}+\left(x_{k, j}^{t}-x_{i, j}^{t}\right) \times F L \times \operatorname{randn}(0,1)
\end{gathered}
$$

Where $\operatorname{randn}(0,1)$ denotes the random number of Gaussian distribution is 0 , the standard deviation is $1 . k(k \neq i) . F L \in(0,2)$ means that the scrounger would follow the producer to find food. We suppose the flight frequency is FQ, Where FQ is a positive integer.

\section{Improved Bird Swarm}

\subsection{Improved Method}

Chaotic optimization initialization population: Hautp [11] and his teams have shown that the optimization performance of the clustering intelligence algorithm is related to the initial population. The bird population algorithm cannot guarantee the diversity of the population because it generates the initial population in a random manner, thus affects the performance of the algorithm. Therefore, we can adopt the chaos optimization algorithm [12] to initialize the population. In this paper, we adopt this method to distribute individuals evenly in search space and improve the optimization ability of the bird swarm algorithm. 
Introducing adaptive inertia weight: The concept of inertia weight was first proposed by Shi and Eberhart [13], which was used to solve the problem of particle explosion and make great progress. Later, the linear decreasing particle swarm optimization algorithm based on the inertia weight $\mathrm{W}$ proposed by Shi in Document [14], which has a profound effect on the study of the inertia weight.

We can add the inertia weight $\mathrm{W}$ into equation (1). The balance between global search and local search is achieved by adjusting the value of $\mathrm{W}$. This method can speed up the convergence and improve the performance of the algorithm.

$$
W=W_{\max } \cdot\left(1-\ln \left(1+t \frac{e-1}{t_{\max }}\right)\right)+W_{\min } \cdot \ln \left(1+t \frac{e-1}{t_{\max }}\right)
$$

Where $W_{\max }=0.9, W_{\min }=0.4, t$ is the current iteration number, $t_{\max }$ is the maximum number of iteration.

Nonlinear adjustment of learning coefficient: $\mathrm{C}$ and $\mathrm{S}$ in the BSA represent cognitive and social coefficients respectively [15]. This approach adaptively adjusts its own cognitive and social experiences. $\mathrm{C}$ and $\mathrm{S}$ are equal at the early foraging stage of birds. Which indicates that "individual" and "group" have the same influence on particle search process. In the later period of foraging, $\mathrm{C}$ takes a small value, $\mathrm{S}$ takes a larger value, which renders local search capabilities strengthened. The updating formula of the learning coefficient for nonlinear adjustment is as follows:

$$
\begin{gathered}
C^{\prime}=(2 \pi)^{-0.5} \cdot e^{\left(-0.5\left(\frac{t}{t_{\max }}\right)^{2}\right)} \\
S^{\prime}=\left(\frac{2}{\pi}\right)^{0.5}-(2 \pi)^{-0.5} \cdot e^{\left(-0.5\left(\frac{t}{t_{\max }}\right)^{2}\right)}
\end{gathered}
$$

The improved foraging formula is as follows:

$$
x_{i, j}^{t+1}=W x_{i, j}^{t}+\left(p_{i, j}-x_{i, j}^{t}\right) \cdot C^{\prime} \cdot \operatorname{rand}(0,1)+\left(g_{j}-x_{i, j}^{t}\right) \cdot S^{\prime} \cdot \operatorname{rand}(0,1)
$$

Producer location added disturbance: Formula (6) is a positional renewal formula for foraging, indicating that the scrounger obtains food from producer. Since the scroungers only search food follow the producer, if the producer falls into a local optimization, they will also fall into a local optimum. In order to solve the problem, the disturbance items is added into the producer's position formula (5), which could enhance its ability to obtain a global optimal solution to avoid local optimization effectively. The improved producer position formula is as follows:

$$
x_{i, j}^{t+1}=x_{i, j}^{t}+\operatorname{randn}(0,1) \cdot x_{i, j}^{t}+\operatorname{randn}(0,1) \cdot\left(\frac{t_{\max }-t+1}{t_{\max }}\right)^{2}
$$

\subsection{IBSA Flow}

Step 1: Initialize each parameter. Setting $\mathrm{N}$ as the total population, setting the initial values of $a_{1}, a_{2}$ and FQ. Defining the spatial dimension of the bird swarm search dim is 2 .

Step 2: Set the maximum iteration number of the outside loop is ${ }^{t_{\max }}$.

Step 3: Set the variable $i=1$, enter the inner loop, and update the location information of the bird group in the foraging behavior according to equation (2), (9), (11), (6).

Step 4: Let $t=t+1$, determine whether the current iteration number reaches FQ. If it does, the birds will be divided into two parts of scrounger and producers. And according to equations (6) and (11) to update the bird group position respectively, otherwise skip equation (6) to continue iterating.

Step 5: If the maximum number of iterations is reached or the bird group position is not updated in a number of iterations, the calculation will stop and the optimal reference value is obtained. 


\section{Numerical Analysis and Experiment}

\subsection{The Test Functions and Experiment}

In order to verify the effectiveness of IBSA in this paper, the following 6 standard test functions [16] are selected for simulation experiment, and the optimization results are compared with the results of the BSA, PSO and GSA respectively. Table 1 is the basic information for each test function. The relevant parameters of the six algorithms are shown in Table 2. For the sake of unity, the population size of each algorithm is 30 , the spatial dimension is 30 , and the maximum number of iterations is 500 .

\subsection{Experimental Results and Analysis}

In order to avoid accidental factors affecting the results, each algorithm runs 30 times. The performance of the algorithm is evaluated through 30 averages results value and standard deviations. It's known to Table3, compared with the BSA, the IBSA has achieved good results in six test functions, and its performance is far better than that of BSA. In addition to function F4, the accuracy of IBSA is much better than that of GSA, and the algorithm stability is improved. In addition to function F6, the accuracy and stability of IBSA are better than PSO. The optimization results on the test function show that in addition to a couple of functions, the IBSA is superior to the other three algorithms in terms of optimization accuracy and algorithm stability.

Table 1. Test Function

\begin{tabular}{ccccc}
\hline Function Name & Test Function & dim & Range & Min \\
\hline $\begin{array}{c}\text { Rosen rock } \\
\text { Quartic }\end{array}$ & $F_{1}(x)=\sum_{i=1}^{19}\left[100\left(x_{i+1}-x_{i}^{2}\right)^{2}+\left(x_{i}-1\right)^{2}\right]$ & 30 & {$[-30,30]$} & 0 \\
$F_{2}(x)=\sum_{i=1}^{n} i x_{i}^{4}+$ random $[0,1)$ & 30 & {$[-1.28,1.28]$} & 0 \\
Penalized1 & $F_{3}(x)=\frac{\pi}{n}\left\{10 \sin ^{2}\left(\pi y_{1}\right)+\sum_{i=1}^{n-1}\left(y_{i}-1\right)^{2}\left[1+10 \sin ^{2}\left(\pi y_{i+1}\right)\right]+\left(y_{n}-1\right)^{2}\right\}$ & 30 & {$[-50,50]$} & 0 \\
& $+\sum_{i=1}^{n} u\left(x_{i}, 10,100,4\right)$ & & \\
Penalized2 & $F_{3}(x)=\frac{\pi}{n}\left\{10 \sin ^{2}\left(\pi y_{1}\right)+\sum_{i=1}^{n-1}\left(y_{i}-1\right)^{2}\left[1+10 \sin ^{2}\left(\pi y_{i+1}\right)\right]+\left(y_{n}-1\right)^{2}\right\}$ & 30 & {$[-50,50]$} & 0 \\
Foxholes & $+\sum_{i=1}^{n} u\left(x_{i}, 10,100,4\right)$ & 2 & {$[-65,65]$} & 0 \\
Kowalik & $F_{5}(x)=\left(\frac{1}{500}+\sum_{j=1}^{25} \frac{1}{j+\sum_{i=1}^{2}\left(x_{i}-a_{i j}\right)^{6}}\right)^{-1}$ & 4 & {$[-5,5]$} & 0.003 \\
\hline
\end{tabular}

Table 2. Related Parameter Setting of 4 Algorithms

\begin{tabular}{cc}
\hline Algorithm & parameter settings \\
\hline IBSA & $c_{1}=1.5, c_{2}=1.5, W_{\max }=0.9, W_{\min }=0.4, a_{1}=a_{2}=1, F Q=10$ \\
BSA & $c_{1}=1.5, c_{2}=1.5, W_{\max }=0.9, W_{\min }=0.4, a_{1}=a_{2}=1, F Q=10$ \\
PSO & $c_{1}=1.5, c_{2}=1.5, W_{\max }=0.9, W_{\min }=0.4, V_{\max }=6$ \\
GSA & $\alpha=20, G_{0}=100$ \\
\hline
\end{tabular}

In order to demonstrate the superiority of the IBSA more intuitively, the convergence characteristic curve of the optimal individual fitness value of the 4 algorithms for 6 benchmark functions are shown in Figure 1. 
Table 3. Test Result

\begin{tabular}{ccccccccc}
\hline \multirow{2}{*}{ Function } & Mean & $\begin{array}{c}\text { IBSA } \\
\text { Standard } \\
\text { Value }\end{array}$ & $\begin{array}{c}\text { Mean } \\
\text { Dalue }\end{array}$ & $\begin{array}{c}\text { BSA } \\
\text { Standard } \\
\text { Deviation }\end{array}$ & $\begin{array}{c}\text { Mean } \\
\text { Value }\end{array}$ & $\begin{array}{c}\text { PSO } \\
\text { Standard } \\
\text { Deviation }\end{array}$ & $\begin{array}{c}\text { Mean } \\
\text { Value }\end{array}$ & $\begin{array}{c}\text { GSA } \\
\text { Standard } \\
\text { Deviation }\end{array}$ \\
\hline \multirow{2}{*}{ F1 } & $8.6595 \mathrm{E}+00$ & $1.3124 \mathrm{E}+01$ & $2.8969 \mathrm{E}+01$ & $2.2874 \mathrm{E}-02$ & $6.9295 \mathrm{E}+01$ & $6.0298 \mathrm{E}+01$ & $8.9761 \mathrm{E}+01$ & $7.4553 \mathrm{E}+01$ \\
F2 & $9.6044 \mathrm{E}-05$ & $9.4087 \mathrm{E}-05$ & $1.7440 \mathrm{E}-04$ & $1.6649 \mathrm{E}-04$ & $7.4070 \mathrm{E}-02$ & $2.4040 \mathrm{E}-02$ & $1.2690 \mathrm{E}-01$ & $4.8616 \mathrm{E}-02$ \\
F3 & $5.4394 \mathrm{E}-02$ & $7.2285 \mathrm{E}-02$ & $1.1803 \mathrm{E}+00$ & $2.5065 \mathrm{E}-01$ & $3.2226 \mathrm{E}+00$ & $1.9091 \mathrm{E}+00$ & $8.0547 \mathrm{E}-02$ & $1.2747 \mathrm{E}-01$ \\
F4 & $1.5222 \mathrm{E}-03$ & $3.1062 \mathrm{E}-03$ & $2.9971 \mathrm{E}+00$ & $2.2518 \mathrm{E}-03$ & $2.4105 \mathrm{E}+01$ & $2.2389 \mathrm{E}+01$ & $1.9844 \mathrm{E}-02$ & $2.8248 \mathrm{E}-02$ \\
F5 & $3.7603 \mathrm{E}+00$ & $2.7593 \mathrm{E}+00$ & $4.5342 \mathrm{E}+00$ & $3.4289 \mathrm{E}+00$ & $6.2556 \mathrm{E}+00$ & $3.7288 \mathrm{E}+00$ & $3.9185 \mathrm{E}+00$ & $2.8933 \mathrm{E}+00$ \\
F6 & $6.4909 \mathrm{E}-04$ & $3.5180 \mathrm{E}-04$ & $7.8034 \mathrm{E}-03$ & $1.0180 \mathrm{E}-02$ & $5.0768 \mathrm{E}-03$ & $3.6392 \mathrm{E}-03$ & $7.3029 \mathrm{E}-04$ & $2.4753 \mathrm{E}-04$ \\
\hline
\end{tabular}

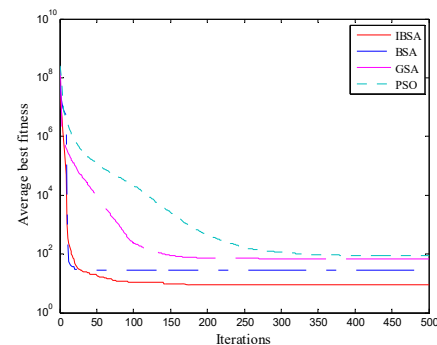

(A)F1

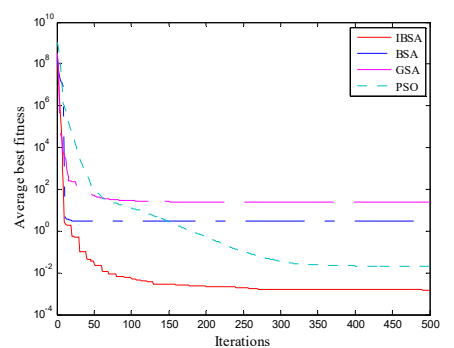

(D)F4

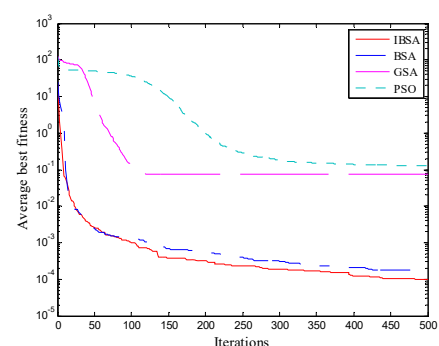

(B)F2

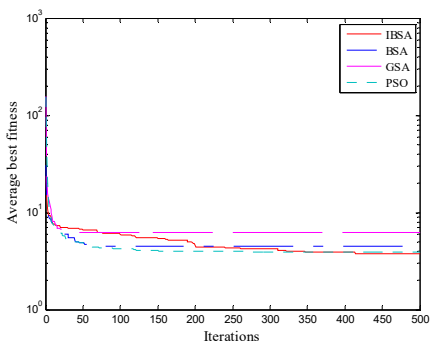

(E)F5

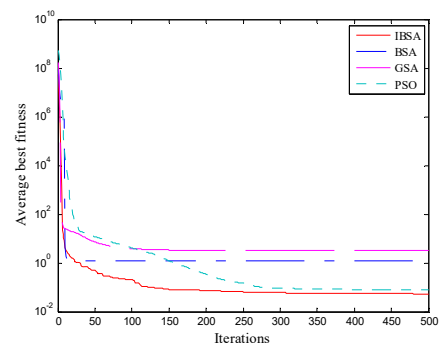

(C)F3

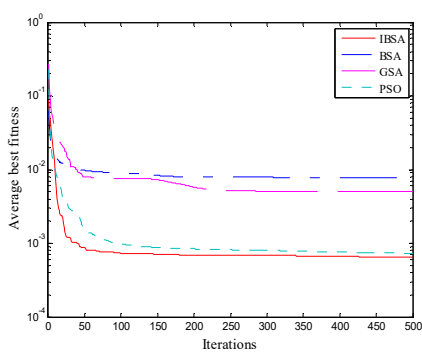

(F)F6

Fig. 1 Target Function Value Curve of 4 Different Algorithms for 6 Benchmark Test Functions

It is known from the convergence curve. Compared with the other three algorithms, the precision and speed of the IBSA for function F4 are obviously improved. For function F3 and F6, IBSA is similar to PSO, but it is superior to BSA and GSA. The optimization results of IBSA are better than those of the other three algorithms for the function F1, F2 and F5. In general, the optimization performance of IBSA is better than the other three algorithms.

\section{Conclusion and Future Work}

In this paper, an adaptive improved bird swarm algorithm is proposed. The IBSA, BSA, PSO and GSA are simulated by adopting 6 benchmark functions, and the experiment result shows that the improved bird swarm algorithm can not only avoid premature convergence but also improve the search accuracy of the algorithm. In the future, the focus of our work is to discuss how to apply the improved bird swarm algorithm to the field of engineering and robot application, combining theory with practice.

\section{References}

[1]. Beheshti Z, Shamsudding S.M.H, A review of population-based meta-heuristic algorithms [J]. International Journal Advance Soft Computer Application, 5(1), 2013, p.1-35. 
[2]. X. Huang, C. M. Ye, J. Zheng, A Chicken-Group Optimization Algorithm Based on Hybrid Improved Search Strategy [J]. Computer Engineering and Applications, 2018, p.1-8.

[3]. Dorigo M, Birattari M, Ant colony optimization [M].Encyclopedia of machine learning. Springer, Boston, MA, 2011, p.36-39.

[4]. Kennedy J, Eberhart R, Particle swarm optimization[C]. Proceedings of the 1995 IEEE International Conference on Neural Networks. Piscataway, NJ, IEEE, 1995, p. 1942-1948.

[5]. Rashedi E, Nezamabadi-pour H, Saryazdi S, GSA: a gravitational search algorithm [J]. Information Sciences, 179(13), 2009, p. 2232-2248.

[6]. X Meng, Y Liu, n X Gao, et al. A New Bio-inspired Algorithm: Chicken Swarm Optimization [M].Advances in Swarm Intelligence. Springer International Publishing, 2014, p.86-94.

[7]. C Zeng, C Peng, K Wang, et al. Multi-objective operation optimization of microgrid based on bird swarm algorithm[J].Power system protection and control, 44(13),2016,p.117-122.

[8]. D Cui, B Jin, Improved bird swarm algorithm and its application in the optimal scheduling of cascade reservoirs [J]. Journal of three Gorges University, 38(6), 2016, p.7-14.

[9]. C Qu, Y Fu, M Luo, Bird Group Algorithm for Solving Flexible Job Shop Scheduling Problem[J],Power system protection and control, 2017.

[10]. J Xue, Y Le, Particle Swarm Optimization algorithm for position perturbation [J]. Computer Engineering and Design, 35(03), 2014, p.1037-1040.

[11]. Haupt R, Haupt S, Practical genetic algorithm [M]. New York: John Wiley and Sons, 2004.

[12]. J Zhang, J Feng, Particle Swarm Optimization based on dynamic chaos perturbation and its Application [J]. Computer Engineering, 2011, p.1517-1520.

[13]. Shi Y, Eberhart R, A modified particle swarm optimizer [M]. Advances in Natural Computation. Springer Berlin Heidelberg, 1998, p.439-439.

[14]. Shi Y H, Eberhart R C. Empirical study of particle swarm optimization [J]. Journal of System Simulation, 1999, 3(1), p.31-37.

[15]. W Wang, Research on particle swarm optimization and its engineering application [D]. Southwest Jiao tong University, 2013.

[16]. C Chen, R Chellali, Y Xin, an improved Grey Wolf Optimization algorithm based on dynamic weights and probabilistic perturbations [J]. Computer Application. 2017, p.34933497. 